\title{
Synthesis and structure study of new manganese and indium based phosphate: $(\mathrm{Ca}, \mathrm{Ba})_{1.222} \mathrm{Mn}_{0.923} \mathrm{In}_{1.570}\left(\mathrm{PO}_{4}\right)_{3}$
}

\author{
Elhassan Benhsina*, Abderrazzak Assani, Mohamed Saadi and Lahcen El Ammari \\ Laboratoire de Chimie Appliquée des Matériaux, Centre des Sciences des Matériaux, Faculty of Sciences, Mohammed V \\ University in Rabat, Avenue Ibn Battouta, BP 1014, Rabat, Morocco. \\ *Correspondence e-mail: h.benhsina@gmail.com
}

\begin{abstract}
Phosphates are an important class of materials which are well-known due to their structural diversity and their important potential application in many fields of sciences. A member of this family, represented by transition metal phosphates, is in the forefront of important researchers. A new manganese and indium based phosphate is successfully isolated by means of solid state reaction. Its corresponding crystal structure is isotypic to the alluaudite and crystallizes in the monoclinic system with the space group $\mathrm{C} 2 / \mathrm{c}$ and cell parameters $\mathrm{a}=12.4697(5) \AA$; $\mathrm{b}=12.9365(5) \AA ; \mathrm{c}=6.5625(3) \AA$ and $\beta=115.603(1)^{\circ}$. The framework of this phosphate is built up on the basis of $\mathrm{MnO}_{6}, \mathrm{InO}_{6}$ and $\mathrm{PO}_{4}$ polyhedra. The $\mathrm{InO}_{6}$ octahedra are linked together via common edge, leading to the formation of $\operatorname{In}_{2} \mathrm{O}_{10}$ dimers. The $\operatorname{In}_{2} \mathrm{O}_{10}$ dimers share edge with $\mathrm{MnO}_{6}$ octahedra, generating chains along the $[10 \overline{1}]$ direction. The $\mathrm{PO}_{4}$ tetrahedra connect two chains giving arise to a three-dimensional framework with hexagonal tunnels where the barium and calcium are located.
\end{abstract}

\section{Introduction}

Les matériaux phosphatés connaissent un développement considérable en raison de leur diversité structurale et de leurs importantes et potentielles applications dans de nombreux domaines de la technologie moderne. Nous pouvons citer, à titre d'exemple, le phosphate $\mathrm{LiFePO}_{4}$ [1] de structure type olivine, dont l'importance est amplement justifiée par son application dans les batteries au lithium commercialisés à l'échelle mondiale. De même, les propriétés électriques de $\mathrm{LiMnPO}_{4}$ [2], de structure alluaudite, avoisinent celles de $\mathrm{LiFePO}_{4}$ et représente l'un des matériaux les plus prometteurs dans le stockage d'énergie en tant que cathode pour les batteries. Par conséquent, l'un des principaux axes de recherche développés dans notre laboratoire est axé sur la synthèse et la caractérisation structurale de nouveaux matériaux phosphatés, apparentés essentiellement à la très convoitée famille alluaudite. Ce choix est justifié par les propriétés physico-chimiques de ces matériaux.
En déployant plusieurs méthodes de synthèse : synthèse par diffusion à l'état solide, synthèse hydrothermale ou synthèse par chimie douce, et en utilisant la diffraction sur monocristal ou sur poudre, nous avons pu isoler et caractériser plusieurs phosphates de type alluaudite. A titre d'exemple, $\mathrm{NaMg}_{3}\left(\mathrm{HPO}_{4}\right)_{2}\left(\mathrm{PO}_{4}\right) \quad$ [3], $\mathrm{AgMg}_{3}\left(\mathrm{HPO}_{4}\right)_{2}\left(\mathrm{PO}_{4}\right)$ [4], $\mathrm{Na}_{2} \mathrm{Co}_{2} \mathrm{Fe}\left(\mathrm{PO}_{4}\right)_{3}[5]$ et $\mathrm{Na}_{1,67} \mathrm{Zn}_{1,67} \mathrm{Fe}_{1,33}\left(\mathrm{PO}_{4}\right)_{3}$ [6].

En effet, la structure du premier phosphate de cette famille alluaudite est déterminée par P.B. Moore [7]. Ces phosphates cristallisent dans un système monoclinique avec un groupe d'espace C2/c. Moore a montré que cette famille peut être représentée par la formule générale $\mathrm{A}(2) \mathrm{A}(1) \mathrm{M}(1) \mathrm{M}(2)_{2}\left(\mathrm{PO}_{4}\right)_{3}$ où $\mathrm{A}$ représente des sites cationiques pouvant être occupés par des cations de moyen ou grande taille et de faible charge (généralement monovalent ou divalent) alors que les sites cationiques de type $M$ représentent un environnement octaédrique généralement occupé par des éléments de transitions. Toutefois, le site de type $\mathrm{M}(1)$ est très distordu alors que celui de type $\mathrm{M}(2)$ est presque régulier. 
Dans ce travail, nous présentons la synthèse et la caractérisation structurale du nouveau phosphate à base d'indium et de manganèse $(\mathrm{Ca}, \mathrm{Ba})_{1,222} \mathrm{Mn}_{0,923} \mathrm{In}_{1,570}\left(\mathrm{PO}_{4}\right)_{3}$.

\section{Expérimental}

Le nouveau phosphate $(\mathrm{Ca}, \mathrm{Ba})_{1,222} \mathrm{Mn}_{0,923} \mathrm{In}_{1,570}\left(\mathrm{PO}_{4}\right)_{3}$ a été obtenu par la réaction de diffusion à l'état solide. Les réactifs (nitrates de calcium (Riedel-de haën, 98\%), nitrate de baryum (Riedel-de haën, 98.5\%), carbonate de manganèse (Riedel-de haën, 99\%), iodure d'indium (Ventron, 98.5) et l'acide phosphorique (Riedel-de haën, $85 \%)$ ) sont dissouts dans l'eau distillée avec quelque goutte d'acide nitrique. Le mélange réactionnel ainsi obtenu est maintenu sous agitation pendant 24 heures avant d'être porté à sec. La poudre obtenue est ensuite broyée dans un mortier en agate et est portée progressivement à la fusion à une température de $1080^{\circ} \mathrm{C}$, dans un creuset en platine. Après un refroidissement lent avec une vitesse de $5^{\circ} \mathrm{C} /$ heure jusqu'à la température ambiante, nous obtenons des cristaux de taille suffisante pour la diffraction des rayons $\mathrm{X}$ sur monocristal.

\section{Résultat et discussions}

\subsection{Collection des données et résolution structurale}

La résolution structurale a été réalisée en utilisant plusieurs réflexions collectées par le diffractomètre à quatre cercle de type Brüker X8 APEX[8] des Unités d'Appui Techniques à la Recherche Scientifique (UATRS) relevant du Centre National pour la recherche Scientifique et Technique CNRST (Maroc). En effet, seules les intensités répondant au critère statistique $\mathrm{I}>2 \sigma(\mathrm{I})(\sigma(\mathrm{I})$ étant l'écart type sur l'intensité) ont été utilisées pour localiser les positions des atomes. Ces données ont été corrigées des effets de Lorentz polarisation et des effets d'absorption. Le tableau 1 montre les conditions d'enregistrement et les données cristallographiques de $(\mathrm{Ca}, \mathrm{Ba})_{1,222} \mathrm{Mn}_{0,923} \mathrm{In}_{1,570}\left(\mathrm{PO}_{4}\right)_{3}$. La résolution et l'affinement de la structure ont été réalisés par la méthode directe couplée aux synthèses de Fourier différences successives, à l'aide des programmes SHELXT2014 [9] et SHELXL2014 [10].

Tableau 1: Données cristallographiques et conditions d'enregistrement de $(\mathrm{Ca}, \mathrm{Ba})_{1,222} \mathrm{Mn}_{0,923} \mathrm{In}_{1,570}\left(\mathrm{PO}_{4}\right)_{3}$.

\begin{tabular}{|c|c|}
\hline \multicolumn{2}{|c|}{ Donnée du cristal } \\
\hline Formule chimique & $(\mathrm{Ca}, \mathrm{Ba})_{1,222} \mathrm{Mn}_{0,923} \mathrm{In}_{1,570}\left(\mathrm{PO}_{4}\right)_{3}$ \\
\hline Système cristallin & Monoclinique \\
\hline Groupe d'espace & $\mathrm{C} 2 / \mathrm{c}$ \\
\hline
\end{tabular}

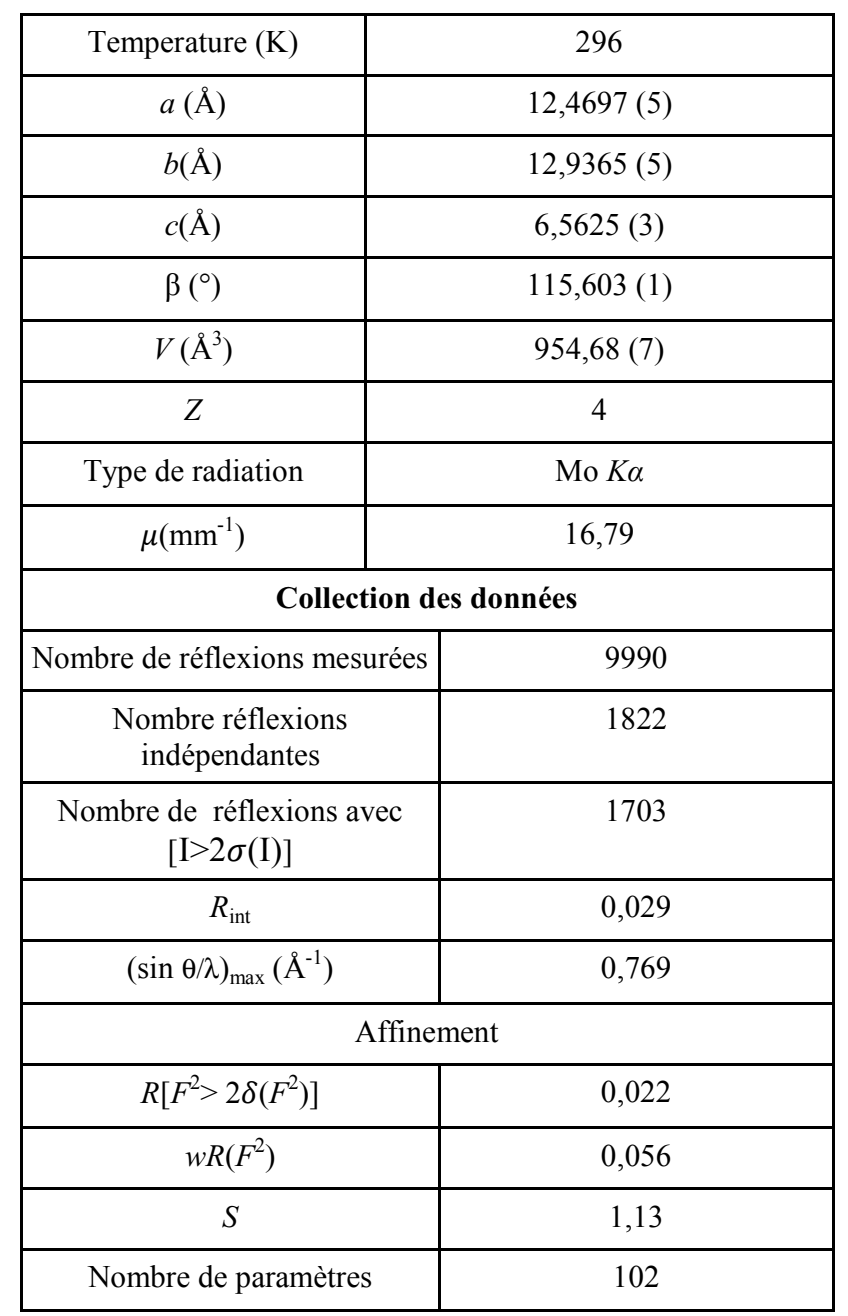

Les conditions d'extinction observées pour $(\mathrm{Ca}, \mathrm{Ba})_{1,222} \mathrm{Mn}_{0,923} \operatorname{In}_{1,570}\left(\mathrm{PO}_{4}\right)_{3}$ sont compatibles avec les groupes d'espace $\mathrm{C} 2 / \mathrm{c}$ et $\mathrm{Cc}$. La structure de ce phosphate a été résolue dans le groupe centro-symétrique (C2/c). Précisons que la méthode des moindres carrés, appliquée sur le carré des modules des facteurs de structure $\mathrm{F}^{2}$, a été utilisée pour affiner les différents paramètres structuraux (positions atomiques $(\mathrm{x}, \mathrm{y}, \mathrm{z}$ ), paramètres de déplacement isotropes $\left(\mathrm{U}_{\text {iso }}\right)$ ou anisotropes $\left(\mathrm{U}_{\text {aniso }}\right)$ et occupation des sites cristallographiques). Les facteurs d'accord, obtenus à la fin du dernier cycle d'affinement, $\mathrm{R}(\mathrm{F})=2,20 \%$, $\mathrm{wR}(\mathrm{F} 2)=5,60 \%$ et $\mathrm{GooF}=$ $\mathrm{S}=1,13$. Ces valeurs attestent de la bonne qualité de l'affinement.

Le tableau 2 regroupe les positions atomiques, les déplacements atomiques isotropes équivalents ainsi que les taux d'occupations des sites cristallographiques. Le tableau 3 rassemble les facteurs de déplacements atomiques anisotropes dans $(\mathrm{Ca}, \mathrm{Ba})_{1,222} \mathrm{Mn}_{0,923} \mathrm{In}_{1,570}\left(\mathrm{PO}_{4}\right)_{3}$. 
Tableau 2: Positions atomiques, déplacements atomiques isotropes équivalents et taux d'occupations des sites cristallographiques dans $(\mathrm{Ca}, \mathrm{Ba})_{1,222} \mathrm{Mn}_{0,923} \mathrm{In}_{1,570}\left(\mathrm{PO}_{4}\right)_{3}$.

\begin{tabular}{|l|l|l|l|l|l|l|}
\hline & Wyck. & $\boldsymbol{x}$ & $\boldsymbol{y}$ & $\boldsymbol{z}$ & $\boldsymbol{U}_{\boldsymbol{~} q}$ & Occ. \\
\hline In1 & $8 \mathrm{f}$ & $0,721(2)$ & $0,3477(1)$ & $0,6327(3)$ & $0,009(1)$ & $0,785(1)$ \\
\hline Mn1 & $4 \mathrm{e}$ & 1,00 & $0,7375(1)$ & 0,750 & $0,010(1)$ & $0,923(2)$ \\
\hline Ca1 & $4 \mathrm{e}$ & 0,500 & $0,4866(1)$ & 0,250 & $0,033(1)$ & $0,922(1)$ \\
\hline Ba1 & $8 \mathrm{f}$ & $0,522(1)$ & $-0,0006(1)$ & $0,466(1)$ & $0,026(1)$ & $0,151(1)$ \\
\hline P1 & $8 \mathrm{f}$ & $0,7397(1)$ & $0,6104(1)$ & $0,6341(1)$ & $0,009(1)$ & 1 \\
\hline P2 & $4 \mathrm{e}$ & 0,5000 & $0,2114(1)$ & 0,2500 & $0,008(1)$ & 1 \\
\hline O1 & $8 \mathrm{f}$ & $0,7263(2)$ & $0,6795(1)$ & $0,8136(2)$ & $0,012(1)$ & 1 \\
\hline O2 & $8 \mathrm{f}$ & $0,6681(2)$ & $0,6628(1)$ & $0,4025(3)$ & $0,014(1)$ & 1 \\
\hline O3 & $8 \mathrm{f}$ & $0,5461(2)$ & $0,2796(1)$ & $0,4621(2)$ & $0,014(1)$ & 1 \\
\hline O4 & $8 \mathrm{f}$ & $0,4069(2)$ & $0,1369(1)$ & $0,2639(4)$ & $0,023(1)$ & 1 \\
\hline O5 & $8 \mathrm{f}$ & $0,6867(2)$ & $0,5042(1)$ & $0,6345(3)$ & $0,019(1)$ & 1 \\
\hline O6 & $8 \mathrm{f}$ & $0,872(2)$ & $0,6031(1)$ & $0,6862(4)$ & $0,024(1)$ & 1 \\
\hline
\end{tabular}

Tableau 3 : Facteurs de déplacements atomiques anisotropes dans le phosphate $(\mathrm{Ca}, \mathrm{Ba})_{1,222} \mathrm{Mn}_{0,923} \mathrm{In}_{1,570}\left(\mathrm{PO}_{4}\right)_{3}$.

\begin{tabular}{|l|l|l|l|l|l|l|}
\hline & $U 11$ & $U 22$ & $U 33$ & $U 12$ & $U 13$ & $U 23$ \\
\hline In1 & $0,01137(9)$ & $0,00810(9)$ & $0,00950(9)$ & $-0,00101(5)$ & $0,00593(6)$ & $-0,00118(5)$ \\
\hline Mn1 & $0,0112(2)$ & $0,0114(2)$ & $0,0107(2)$ & 0,000 & $0,00719(2)$ & 0,000 \\
\hline Ca1 & $0,0148(4)$ & $0,0279(5)$ & $0,0400(6)$ & 0,000 & $-0,0036(3)$ & 0,000 \\
\hline Ba1 & $0,0258(6)$ & $0,0121(4)$ & $0,0267(6)$ & $0,0006(4)$ & $-0,0013(4)$ & $0,0012(4)$ \\
\hline P1 & $0,0135(2)$ & $0,0064(2)$ & $0,0081(2)$ & $0,00066(2)$ & $0,00576(2)$ & $0,00012(2)$ \\
\hline P2 & $0,0078(3)$ & $0,0095(3)$ & $0,0074(3)$ & 0,000 & $0,0031(2)$ & 0,000 \\
\hline O1 & $0,0175(7)$ & $0,0109(6)$ & $0,0083(6)$ & $-0,0002(5)$ & $0,0070(5)$ & $-0,0016(5)$ \\
\hline O2 & $0,0252(8)$ & $0,0104(6)$ & $0,0080(6)$ & $0,0017(5)$ & $0,0081(6)$ & $0,0019(5)$ \\
\hline O3 & $0,0120(6)$ & $0,0211(8)$ & $0,0066(6)$ & $0,0015(5)$ & $0,0027(5)$ & $-0,0030(5)$ \\
\hline O4 & $0,0104(7)$ & $0,0180(8)$ & $0,0394(2)$ & $0,0009(6)$ & $0,0085(7)$ & $0,0145(7)$ \\
\hline O5 & $0,0289(9)$ & $0,0078(7)$ & $0,0242(8)$ & $-0,0004(6)$ & $0,0150(7)$ & $0,0024(6)$ \\
\hline O6 & $0,0170(7)$ & $0,0175(8)$ & $0,0412(2)$ & $0,0021(6)$ & $0,0164(8)$ & $-0,0042(7)$ \\
\hline
\end{tabular}


Les atomes d'indium et de baryum occupent partiellement les sites $8 \mathrm{f}$ avec des taux d'occupation de 0,785 (1) et 0,151 (1) respectivement. Quant aux atomes de manganèse et de calcium, ils occupent partiellement les sites 4e avec des taux d'occupation de 0,993(2) et $0,922(1)$ respectivement. Les autres atomes (phosphore et oxygène) occupent totalement les sites $8 \mathrm{f}$ à l'exception de P2 qui est localisé sur le site 4e. Ceci conduit à la formulation suivante :
$\mathrm{Ca}_{0,922} \mathrm{Ba}_{0,300} \mathrm{Mn}_{0,923} \mathrm{In}_{1,570}\left(\mathrm{PO}_{4}\right)_{3}$. Ce type de nonstœchiométrie est souvent rencontré dans les structures de type alluaudite : $\quad \mathrm{Na}_{1,72} \mathrm{Mn}_{3,28}\left(\mathrm{AsO}_{4}\right)_{3}[11]$, $\mathrm{Ag}_{1,49} \mathrm{Mn}_{2,90}\left(\mathrm{AsO}_{4}\right)_{3}[12], \quad \mathrm{Na}_{1,67} \mathrm{Mn}_{2,17}\left(\mathrm{MoO}_{4}\right)_{3}[13]$, $\mathrm{Na}_{2,44} \mathrm{Mn}_{1,79}\left(\mathrm{SO}_{4}\right)_{3}[14]$.

\section{Description de la structure}

Les distances et les angles significatifs dans le phosphate $(\mathrm{Ca}, \mathrm{Ba})_{1,222} \mathrm{Mn}_{0,923} \operatorname{In}_{1,570}\left(\mathrm{PO}_{4}\right)_{3}$ sont regroupés dans le tableau 4.

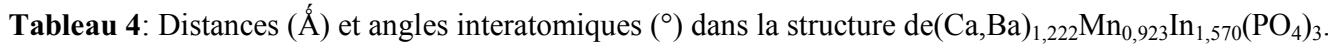

\begin{tabular}{|c|c|c|c|}
\hline Distances P(1) - O (Á) & & Distances P(2) - O (Á) & \\
\hline $\mathrm{P} 1-\mathrm{O} 5$ & $1,5252(2)$ & $\mathrm{P} 2-\mathrm{O} 3^{\mathrm{xii}}$ & $1,5344(2)$ \\
\hline $\mathrm{P} 1-\mathrm{O} 6$ & $1,5355(2)$ & $\mathrm{P} 2-\mathrm{O} 3$ & $1,5345(2)$ \\
\hline $\mathrm{P} 1-\mathrm{O} 2$ & $1,5472(2)$ & $\mathrm{P} 2-\mathrm{O} 4$ & $1,5410(2)$ \\
\hline $\mathrm{P} 1-\mathrm{O} 1$ & $1,5479(2)$ & $\mathrm{P} 2-\mathrm{O} 4^{\mathrm{xii}}$ & $1,5411(2)$ \\
\hline Distance moyenne & $1,5389 \AA$ & Distance moyenne & $1,5376 \AA$ \\
\hline Angles $O$ - P(1) - $O\left({ }^{\circ}\right)$ & & Angles $\mathrm{O}$ - P(2) - O $\left(^{\circ}\right)$ & \\
\hline $\mathrm{O} 5-\mathrm{P} 1-\mathrm{O} 6$ & $111,68(11)$ & $\mathrm{O} 3^{\mathrm{xii}}-\mathrm{P} 2-\mathrm{O} 3$ & $154,72(7)$ \\
\hline $\mathrm{O} 5-\mathrm{P} 1-\mathrm{O} 2$ & $109,24(10)$ & $\mathrm{O} 3^{\mathrm{xii}-\mathrm{P} 2-\mathrm{O} 4}$ & $90,36(6)$ \\
\hline $\mathrm{O} 6-\mathrm{P} 1-\mathrm{O} 2$ & $110,74(11)$ & $\mathrm{O} 3-\mathrm{P} 2-\mathrm{O} 4$ & $109,79(13)$ \\
\hline $\mathrm{O} 5-\mathrm{P} 1-\mathrm{O} 1$ & $109,08(10)$ & $\mathrm{O} 3^{\mathrm{xii}}-\mathrm{P} 2-\mathrm{O} 4^{\mathrm{xii}}$ & $114,63(9)$ \\
\hline $\mathrm{O} 6-\mathrm{P} 1-\mathrm{O} 1$ & $109,27(10)$ & $\mathrm{O} 3-\mathrm{P} 2-\mathrm{O} 4^{\mathrm{xii}}$ & $107,58(11)$ \\
\hline $\mathrm{O} 2-\mathrm{P} 1-\mathrm{O} 1$ & $106,69(9)$ & $\mathrm{O} 4-\mathrm{P} 2-\mathrm{O} 4^{\mathrm{xii}}$ & $102,65(15)$ \\
\hline Distances In - O (Á) & & Distances Mn - O (Á) & \\
\hline $\mathrm{In} 1-\mathrm{O} 5$ & $2,0694(2)$ & $\mathrm{Mn} 1-\mathrm{O}^{\mathrm{vi}}$ & $2,2660(2)$ \\
\hline $\mathrm{In} 1-\mathrm{O} 4^{\mathrm{i}}$ & $2,1073(2)$ & $\mathrm{Mn} 1-\mathrm{O}^{\mathrm{viii}}$ & $2,2660(2)$ \\
\hline $\mathrm{In} 1-\mathrm{O} 2^{\mathrm{ii}}$ & $2,1424(2)$ & $\mathrm{Mn} 1-\mathrm{O}^{\mathrm{ix}}$ & $2,2734(2)$ \\
\hline $\mathrm{In} 1-\mathrm{O} 1^{\mathrm{iii}}$ & $2,1501(2)$ & $\mathrm{Mn} 1-\mathrm{O} 6$ & $2,2735(2)$ \\
\hline $\mathrm{In} 1-\mathrm{O} 3$ & $2,1605(2)$ & $\mathrm{Mn} 1-\mathrm{O} 2^{\mathrm{x}}$ & $2,2905(2)$ \\
\hline $\mathrm{In} 1-\mathrm{O} 1^{\mathrm{iv}}$ & $2,2591(2)$ & $\mathrm{Mn} 1-\mathrm{O} 2^{\mathrm{xi}}$ & $2,2766(2)$ \\
\hline Distance moyenne & $2,1481 \AA$ & Distance moyenne & $2,3434 \AA$ \\
\hline Angles $\mathbf{O}-\mathbf{I n}-\mathbf{O}\left(^{\circ}\right)$ & & Angles $\mathbf{O}-\mathbf{M n}-\mathbf{O}\left(^{\circ}\right)$ & \\
\hline $\mathrm{O} 5-\mathrm{In} 1-\mathrm{O} 4^{\mathrm{i}}$ & $95,63(7)$ & $\mathrm{O} 3^{\mathrm{vi}}-\mathrm{Mn} 1-\mathrm{O} 3^{\mathrm{viii}}$ & $152,22(9)$ \\
\hline $\mathrm{O} 5-\mathrm{In} 1-\mathrm{O} 2^{\mathrm{ii}}$ & $85,41(7)$ & $\mathrm{O} 3^{\mathrm{vi}}-\mathrm{Mn} 1-\mathrm{O}^{\mathrm{ix}}$ & $116,17(7)$ \\
\hline $\mathrm{O} 4^{\mathrm{i}}-\mathrm{In} 1-\mathrm{O} 2^{\mathrm{ii}}$ & $110,19(8)$ & $\mathrm{O} 3^{\text {viii }}-\mathrm{Mn} 1-\mathrm{O}^{\mathrm{ix}}$ & $85,77(7)$ \\
\hline $\mathrm{O} 5-\mathrm{In} 1-\mathrm{O} 1^{\mathrm{iii}}$ & $104,96(7)$ & $\mathrm{O} 3^{\mathrm{vi}}-\mathrm{Mn} 1-\mathrm{O} 6$ & $85,77(7)$ \\
\hline 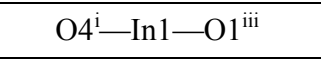 & $85,63(7)$ & $\mathrm{O}^{\text {viii }}-\mathrm{Mn} 1-\mathrm{O} 6$ & $116,17(7)$ \\
\hline $\mathrm{O} 2^{\mathrm{ii}}-\mathrm{In} 1-\mathrm{O} 1^{\mathrm{iii}}$ & $160,45(6)$ & $\mathrm{O} 6^{\mathrm{ix}}-\mathrm{Mn} 1-\mathrm{O} 6$ & $80,23(9)$ \\
\hline $\mathrm{O} 5-\mathrm{In} 1-\mathrm{O} 3$ & $103,80(7)$ & $\mathrm{O} 3^{\mathrm{vi}}-\mathrm{Mn} 1-\mathrm{O} 2^{\mathrm{x}}$ & $90,83(6)$ \\
\hline $\mathrm{O} 4-\mathrm{I} 1-\mathrm{O} 3$ & $159,40(6)$ & $\mathrm{O} 3^{\mathrm{viii}}-\mathrm{Mn} 1-\mathrm{O} 2^{\mathrm{x}}$ & $73,45(6)$ \\
\hline
\end{tabular}




\begin{tabular}{|c|c|c|c|}
\hline $\mathrm{O} 2^{\mathrm{ii}-\mathrm{In} 1-\mathrm{O} 3}$ & $78,58(6)$ & $\mathrm{O} 6^{\mathrm{ix}}-\mathrm{Mn} 1-\mathrm{O} 2^{\mathrm{x}}$ & $85,01(6)$ \\
\hline $\mathrm{O} 1^{\mathrm{iii}-\mathrm{In} 1-\mathrm{O} 3}$ & $82,82(6)$ & $\mathrm{O} 6-\mathrm{Mn} 1-\mathrm{O} 2^{\mathrm{x}}$ & $161,49(7)$ \\
\hline $\mathrm{O} 5-\mathrm{In} 1-\mathrm{O} 1^{\mathrm{iv}}$ & $171,49(7)$ & $\mathrm{O} 3^{\mathrm{vi}}-\mathrm{Mn} 1-\mathrm{O} 2^{\mathrm{xi}}$ & $73,45(6)$ \\
\hline $\mathrm{O} 4^{\mathrm{i}}-\mathrm{In} 1-\mathrm{O} 1^{\mathrm{iv}}$ & $80,08(6)$ & $\mathrm{O} 3^{\mathrm{viii}}-\mathrm{Mn} 1-\mathrm{O} 2^{\mathrm{xi}}$ & $90,83(6)$ \\
\hline $\mathrm{O} 2^{\mathrm{ii}}-\mathrm{In} 1-\mathrm{O} 1^{\mathrm{iv}}$ & $89,18(6)$ & $\mathrm{O}^{\mathrm{ix}}-\mathrm{Mn} 1-\mathrm{O} 2^{\mathrm{xi}}$ & $161,49(7)$ \\
\hline 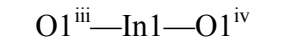 & $82,15(6)$ & $\mathrm{O} 6-\mathrm{Mn} 1-\mathrm{O} 2^{\mathrm{xi}}$ & $85,01(6)$ \\
\hline $\mathrm{O} 3-\mathrm{In} 1-\mathrm{O} 1^{\mathrm{iv}}$ & $81,49(6)$ & $\mathrm{O} 2^{\mathrm{x}}-\mathrm{Mn} 1-\mathrm{O} 2^{\mathrm{xi}}$ & $111,45(8)$ \\
\hline Distances Ca - O (Á) & & Distances Ba- O (Á) & \\
\hline $\mathrm{Ca} 1-\mathrm{O} 5$ & $2,6005(2)$ & $\mathrm{Ba} 1-\mathrm{O} 4$ & $2,308(2)$ \\
\hline $\mathrm{Ca} 1-\mathrm{O}^{\mathrm{xii}}$ & $2,6006(2)$ & $\mathrm{Ba} 1-\mathrm{O} 6^{\mathrm{xv}}$ & $2,377(2)$ \\
\hline $\mathrm{Ca} 1-\mathrm{O} 5^{\mathrm{iii}}$ & $2,7446(2)$ & $\mathrm{Ba} 1-\mathrm{O}^{\mathrm{v}}$ & $2,381(2)$ \\
\hline $\mathrm{Ca} 1-\mathrm{O}^{\mathrm{vii}}$ & $2,7446(2)$ & $\mathrm{Ba} 1-\mathrm{O} 4^{\text {xiv }}$ & $2,384(2)$ \\
\hline $\mathrm{Ca} 1-\mathrm{O} 3$ & $2,9577(2)$ & $\mathrm{Ba} 1-\mathrm{O}^{\mathrm{iv}}$ & $2,480(2)$ \\
\hline $\mathrm{Ca} 1-\mathrm{O}^{\mathrm{xii}}$ & $2,958(2)$ & $\mathrm{Ba}-\mathrm{O}^{\mathrm{iiv}}$ & 2,7204 \\
\hline $\mathrm{Ca} 1-\mathrm{O} 2$ & $2,9623(2)$ & Distance moyenne & $2,4417 \AA$ \\
\hline $\mathrm{Ca} 1-\mathrm{O} 2^{\mathrm{xii}}$ & $2,9623(2)$ & & \\
\hline Distance moyenne & $2,8163 \AA$ & & \\
\hline
\end{tabular}

Codes de symétrie : (i) $x+1 / 2,-y+1 / 2, z+1 / 2$; (ii) $x,-y+1, z+1 / 2$; (iii) $x,-y+1, z-1 / 2$; (iv) $-x+3 / 2, y-1 / 2,-z+3 / 2$; (v) $-x+3 / 2,-y+1 / 2$, $z+1$; (vi) $-x+3 / 2, y+1 / 2,-z+3 / 2$; (vii) $-x+1,-y+1,-z+1$; (viii) $x+1 / 2, y+1 / 2, z$; (ix) $-x+2, y,-z+3 / 2$; (x) $x+1 / 2,-y+3 / 2, z+1 / 2$; (xi) $x+3 / 2,-y+3 / 2,-z+1$; (xii) $-x+1, y,-z+1 / 2$; (xiii) $-x+1,-y+1,-z$; (xiv) $-x+1,-y,-z+1$; (xv) $x-1 / 2,-y+1 / 2, z-1 / 2$; (xvi) $x-1 / 2, y-1 / 2, z$; (xvii) $x,-y, z-1 / 2$; (xviii) $x,-y, z+1 / 2$.

La structure de ce phosphate est formée de tétraèdres $\mathrm{PO}_{4}$, d'octaèdres $\mathrm{InO}_{6}$ et $\mathrm{MnO}_{6}$.

Deux octaèdres d'indium mettent des arêtes en commun pour former un dimer $\operatorname{In}_{2} \mathrm{O}_{10}$. Ces derniers sont liés alternativement par les octaèdres de manganèse $\mathrm{MnO}_{6}$ pour construire des chaînes parallèle à la

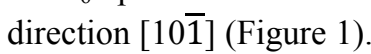

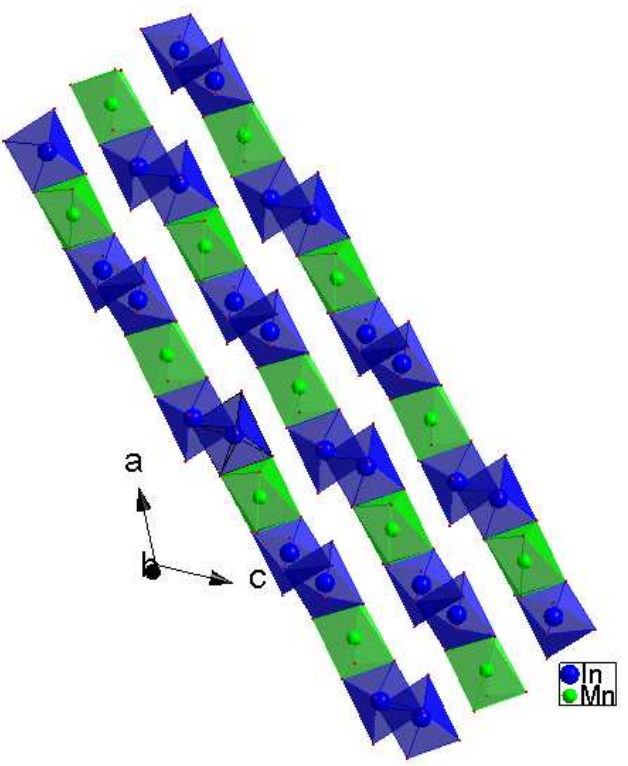

Figure 1: Chaînes formées par les octaèdres $\mathrm{MnO}_{6}$ et $\mathrm{InO}_{6}$ parallèle à $[10 \overline{1}]$.
Ces chaînes sont connectées via les sommets des tétraèdres $\mathrm{P}_{10}$ et $\mathrm{P}_{2} \mathrm{O}_{4}$, pour former une charpente tridimensionnelle délimitant des tunnels dans lesquels les atomes de baryum et de calcium se logent (Figure 2).

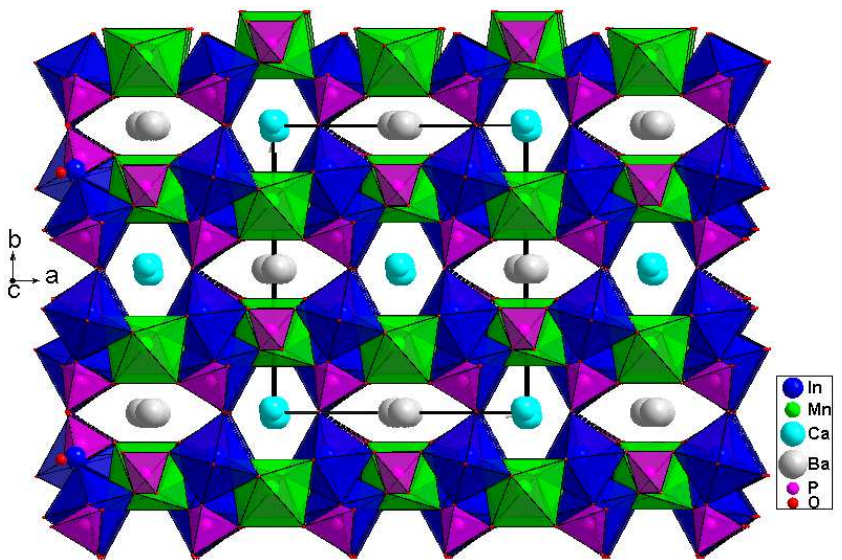

Figure 2 : $\quad$ Projection de la structure $(\mathrm{Ca}, \mathrm{Ba})_{1,222} \mathrm{Mn}_{0,923} \mathrm{In}_{1,570}\left(\mathrm{PO}_{4}\right)_{3}$ selon l'axe c.

\section{Remerciements :}

Les auteurs remercient les Unités d'Appui Techniques à la Recherche Scientifique (UATRS), relevant du Centre National pour la recherche Scientifique et Technique (CNRST) (Maroc) pour la collecte des données de la diffraction de rayons X. 


\section{Références}

1. L.-X. Yuan et al., Energy Environ. Sci., vol. 4, no. 2, pp. 269-284, (2011).

2. J. Kim, H. Kim, K. Park, Y. Park, and S. Lee, vol. 4, pp. 8632-8636, (2014).

3. A. Assani, M. Saadi, M. Zriouil, .\& L. El Ammari, Acta Cryst. E67, i5 (2011a).

4. A. Ould Saleck, A. Assani, M. Saadi, C. Mercier, C. Follet, and L. El Ammari, Acta Crystallogr. Sect. E Crystallogr. Commun., vol. 71, no. 7, pp. 813-815, (2015).

5. A. Bouraima, A. Assani, M. Saadi, T. Makani, and L. El Ammari, Acta Crystallogr. Sect. E Crystallogr. Commun., vol. 71, no. Ii, pp. 558-560, (2015).

6. J. Khmiyas, A. Assani, M. Saadi, and L. El Ammari, Acta Crystallogr. Sect. E Crystallogr. Commun., vol. 71, no. Iii, pp. 690-692, (2015).

7. P. B. Moore, Am. Mineral., vol. 56, p. 1955, (1971).

8. Bruker APEX2 and SAINT. Bruker AXS Inc., Madison, Wisconsin, USA. (2005).

9. G. M. Sheldrick, Acta Cryst.A71, 3-8 (2015a).

10. G. M. Sheldrick, Acta Cryst.C71, 3-8 (2015b).

11. B. Ayed, M. Krifa \& A. Haddad Acta Cryst. C58, i58, i98-100 (2002).

12. B. Ayed \& A. Haddad, Acta Cryst E59, i77-i79 (2003).

13. C. Bouzidi, M. Faouzi Zid, A. Driss \& W. Frigui. Acta Cryst. E70, i18-i19 (2014).

14. Debasmita Dwibedi, Rafael B. Araujo, Sudip Chakraborty, Pradeep P. Shanbogh, alini G. Sundaram, Rajeev Ahuja, \& Prabeer Barpanda J. Mater. Chem. A, (2015). 